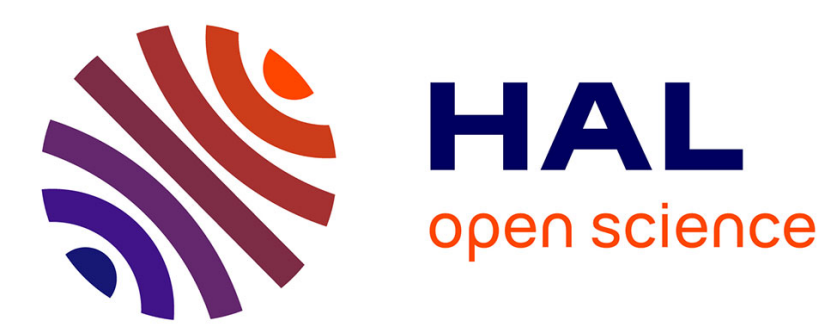

\title{
Simulation of transient Rayleigh-Bénard-Marangoni convection induced by evaporation
}

O. Touazi, Eric Chénier, F. Doumenc, B. Guerrier

\section{To cite this version:}

O. Touazi, Eric Chénier, F. Doumenc, B. Guerrier. Simulation of transient Rayleigh-BénardMarangoni convection induced by evaporation. International Journal of Heat and Mass Transfer, 2010, 53 (4), pp.656-664. 10.1016/j.ijheatmasstransfer.2009.10.029 . hal-00691272

\section{HAL Id: hal-00691272 \\ https://hal.science/hal-00691272}

Submitted on 25 Apr 2012

HAL is a multi-disciplinary open access archive for the deposit and dissemination of scientific research documents, whether they are published or not. The documents may come from teaching and research institutions in France or abroad, or from public or private research centers.
L'archive ouverte pluridisciplinaire HAL, est destinée au dépôt et à la diffusion de documents scientifiques de niveau recherche, publiés ou non, émanant des établissements d'enseignement et de recherche français ou étrangers, des laboratoires publics ou privés. 


\title{
Simulation of transient Rayleigh-Bénard-Marangoni convection induced by evaporation
}

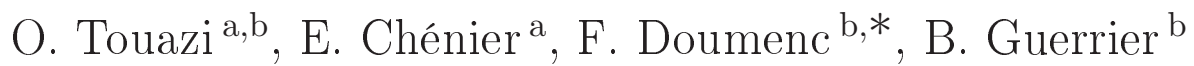 \\ ${ }^{a}$ Université Paris-Est, laboratoire Modélisation et Simulation Multi Echelle, \\ MSME FRE3160 CNRS, 5 bd Descartes, 77454 Marne-la-Vallee, France \\ b Univ Pierre et Marie Curie-Paris6, Univ Paris-Sud, CNRS, F-91405, \\ Lab FAST, Bat 502, Campus Univ, Orsay, F-91405.
}

\begin{abstract}
Numerical simulation of thermal convection induced by solvent evaporation in an initially isothermal fluid is considered. Both thermocapillarity and buoyancy driving forces are taken into account, and a criterium based on the Peclet number is used to analyze the stability of this transient problem. Critical Marangoni and Rayleigh numbers are obtained for a large range of Biot and Prandtl numbers. Results of the non linear simulations are compared with a previous linear transient stability analysis based on a non normal approach and with visualizations performed during PIB/Toluene solutions drying experiments. A scaling analysis is developed for the Marangoni problem and correlations are derived to predict the order of magnitude of temperature and velocity as a function of $B i, M a$ and $P r$ numbers.
\end{abstract}

Key words: free convection, evaporation, stability, heat transfer

\section{Introduction}

Evaporation of a volatil fluid or drying of a solution induces a decrease of the temperature at the free surface due to the vaporization latent heat. This situation can generate convective motion due to both buoyancy and thermocapillary driving forces [1-6]. Several points may be pointed out when studying such

\footnotetext{
* Corresponding author. Tel: (33) 01691537 31; Fax: (33) 0169158060

Email addresses: Eric.Chenier@univ-mlv.fr (E. Chénier), doumenc@fast.u-psud.fr (F. Doumenc), guerrier@fast.u-psud.fr (B. Guerrier).
} 
systems. First the boundary condition at the free surface results from the coupling between the system and the surroundings. The evaporating flux and then the temperature gradient in the fluid depends on the heat and mass transfers with the ambient air. Several authors have developed numerical or theoretical studies taking into account this coupling, for example Merkt and Bestehorn [7], Colinet and co-authors [8], Ozen and Narayanan [9] and Moussy and coauthors [10]. Both surface tension- and buoyancy-driven convection may occur as studied for example by Medale and Cerisier in several geometries but in a nonvolatile fluid [11]. Another point that is more specifically considered in this paper is the transient nature of many experiments. Indeed, starting from an initial state where the fluid is isotherm, evaporation induces a decrease of the surface temperature. The basic temperature field corresponding to the pure diffusive problem (null velocity field) is time dependent so that a classical stability analysis based on the perturbation of a steady conductive regime is questionable. Then the prediction of critical conditions for the onset of convection is a complex problem. A theoretical linear analysis based on a non normal approach has been recently developed to take into account the time dependent basic state for this same problem (cf. [12] and references herein).

In this paper we present a 2D non linear numerical simulation to study the development and evolution of convective patterns in the transient regime, when both buoyancy and thermocapillary effects can be put forward. A large range of parameters characterizing the problem (Biot, Marangoni, Prandtl and Rayleigh numbers) are investigated. This numerical approach follows an experimental study where numerous experiments of drying of a polymer/solvent solution have been performed with different thicknesses and viscosities. Simplifying assumptions follow from experimental results and are presented in section 2, as well as the model equations and numerical method. Section 3 and 4 are devoted to 2D non linear numerical simulations and to the determination of convection thresholds for this transient problem. Comparisons are made with experimental results and with previous results deduced from the linear stability analysis. At last, a scaling analysis is developed for the Marangoni problem in section 5.

\section{Problem formulation}

\subsection{Thermal model}

Drying experiments that underlie the simulations presented in this paper have been performed on the system Polyisobutylene (PIB)/Toluene. Experiments are described in detail in [13] and we only recall the main points used to establish the simplified model considered here. The solution initially at the ambient 
temperature is poured in a dish located in an extractor hood. The two control parameters used in the experiments are the initial thickness $(0.3 \mathrm{~mm} \leq$ $e \leq 14.3 \mathrm{~mm})$ and the initial polymer mass fraction $\left(0 \leq \omega_{P} \leq 15 \%\right)$. Polymer solution viscosity is strongly sensitive to polymer concentration [14]. The viscosity $\mu$ is $5.5 \times 10^{-4} \mathrm{~Pa}$.s and $2.4 \mathrm{~Pa}$.s for $\omega_{P} 0 \%$ and $15 \%$ respectively.

Several simplifying assumptions have been inferred from experimental observations. They are valid only at the beginning of the drying which is the time under consideration in this paper. When evaporation begins, convective patterns have been observed at the very beginning of the experiment(quasiinstantaneous or less than $100 \mathrm{~s}$ after pouring the solution). They disappear well before the end of the drying. The Lewis number (ratio of the thermal diffusivity to mass diffusivity) being very large (about $10^{3}$ ), it is assumed that convective patterns observed in the first minutes are mainly driven by thermal effects. Two experimental observations detailed in [13] support this thesis. First, a few experiments were conducted with deuterated solvent, whose density is higher than the polymer density. In that case, the density of the solution decreases when the polymer concentration increases, leading to a stable situation if the solutal Rayleigh-Bénard problem is considered. Since no differences were found with the experiments conducted with the standard solvent, we can exclude solutal buoyancy as a dominant mechanism. Second, free surface temperature fields measured by infrared camera showed that the end of free convection was related to the duration of the transient thermal regime. Solutal convection is then not taken into account in this paper.

Moreover, the layer thickness is assumed to remain constant. This hypothesis can be adopted if $P e_{i n t} \ll 1$, where the interface Peclet number is defined as $P e_{\text {int }} \equiv\left(e v_{e v}\right) / \alpha$ with $e$ the layer thickness, $v_{e v}$ the interface velocity due to evaporation and $\alpha$ the thermal diffusivity. Indeed when $P e \ll 1$, the surface displacement $v_{e v} \delta_{\text {diff }}$ remains negligible compared to the total thickness $e$ during the problem characteristic time i.e. the diffusion time $\delta_{\text {diff }} \equiv e^{2} / \kappa$. In the experiments, the Peclet number is smaller than 0.1. In the same way, the rate of change of the polymer mass fraction is small and the physical properties of the solution are assumed constant.

The free surface is assumed flat. Surface deformation can be neglected if the crispation number $C r \equiv(\rho \nu \alpha) /(\gamma e) \ll 1[15]$ and if the Galileo number $G a \equiv$ $\left(g e^{3}\right) /(\nu \alpha) \gg 1$, where $\rho, \nu, \gamma$, respectively denote fluid density, viscosity, surface tension, and where $\mathrm{g}$ is the acceleration due to gravity. Such conditions are shown to be satisfied for the experiments considered here. Then surface deformability can be disregarded.

For sake of simplicity the numerical analysis is restricted to a two-dimensional geometry since the study focuses on the onset of convection and not on the description of convective patterns morphology. Comparison of experimental 
and simulated thresholds (see section 4.2) validates the 2D approach a posteriori. The solution layer is contained in a rectangular domain of aspect ratio $A=L / e=20$, where $L$ is the horizontal length and $e$ the thickness of the solution layer. The vertical and bottom solid walls are supposed to be adiabatic and non permeable. Evaporation occurs at the free surface only. Heat and mass transfers between the free surface and the ambient air are described by global transfer coefficients.

With the Boussinesq approximation, the Navier-Stokes and energy equations are:

$$
\begin{aligned}
\vec{\nabla} \cdot \vec{v} & =0, \\
\frac{\partial}{\partial t} \vec{v}+(\vec{v} \cdot \vec{\nabla}) \vec{v} & =-\frac{1}{\rho} \vec{\nabla} p+\nu \Delta \vec{v}+g \beta_{T}\left(T-T_{0}\right) \overrightarrow{e_{y}} \\
\frac{\partial}{\partial t} T+(\vec{v} \cdot \vec{\nabla}) T & =\alpha \Delta T,
\end{aligned}
$$

where $\vec{v}(\vec{x}, t)$ is the velocity field, $p(\vec{x}, t)$ is the pressure field, $T(\vec{x}, t)$ is the temperature field, $T_{0}$ the temperature of the ambient air and $\overrightarrow{e_{y}}$ the unit vector in the vertical direction. In this approximation, the density $\rho$ is taken to be the density at $T=T_{0} . \beta_{T}, \nu=\mu / \rho, \alpha=k / \rho c$ are the thermal expansion coefficient, the kinematic viscosity and the thermal diffusivity with $\mu$ the dynamic viscosity, $k$ the thermal conductivity and $c$ the specific heat.

The polymer mass fraction being small, we have used the physical properties of the toluene except for the viscosity that is very sensitive to polymer concentration. The following values have been used: $\rho=865 \mathrm{~kg} \cdot \mathrm{m}^{-3}$, $k=0.142 W \cdot m^{-1} \cdot K^{-1}, \alpha=0.97 \times 10^{-7} m^{2} \cdot s^{-1}, \beta_{T}=1.07 \times 10^{-3} K^{-1}$. For each experiment the viscosity is also assumed constant but depends on the initial polymer concentration, according to an empirical law (Fig.1) deduced from viscosity measurements performed with a Low Shear 30 rheometer (coaxial cylinders and imposed deformation) [16].

\subsection{Initial and boundary conditions}

At $t=0$ the fluid is at the ambient temperature $T(\vec{x}, 0)=T_{0}$. To study the stability of the system, a perturbation is imposed on the velocity field at $t=0$. Since the structures of real experimental perturbations are not known a priori, a random velocity perturbation with zero mean and amplitude $r$ is implemented in the following way: numerical resolution of the problem is achieved using a finite volume scheme. For each spatial node, the value allotted to the velocity at $t=0$ comes from a random drawing of a uniform distribution between $-r / 2$ and $+r / 2$. Further studies have shown that the choice of the 
initial perturbation does not modify much the stability thresholds (cf. section 3.2 and $[12,17])$.

The solid walls are adiabatic with a zero velocity boundary condition. On the free surface located at $y=e$, the thermal boundary condition is :

$$
-k\left(\frac{\partial T}{\partial y}\right)_{y=e}=h_{t h}\left(T(x, e, t)-T_{0}\right)+L \Phi_{e v}
$$

The first term of the r.h.s. is the convective heat transfer between the free surface and the ambient air where $h_{t h}$ is the heat transfer coefficient. The second term, $L \Phi_{e v}$, corresponds to the solvent evaporation with $L$ the latent heat of vaporization and $\Phi_{e v}$ the evaporative flux, that can be expressed as

$$
\Phi_{e v}=h_{m}\left(\rho_{\text {Sint }}^{g}-\rho_{S \infty}^{g}\right)
$$

where $h_{m}$ is the mass transfer coefficient, $\rho_{\text {Sint }}^{g}$ and $\rho_{S \infty}^{g}$ are the solvent concentration in the gas phase just above the interface and far from the interface respectively. The last one is zero, due to the important air flow rate in the extractor hood. With the ideal gas law, we get:

$$
\Phi_{e v}=h_{m} \frac{M_{S} P_{V S 0}(T(x, e, t))}{R T(x, e, t)} a\left(T(x, e, t), \varphi_{S}(x, e, t)\right)
$$

where $M_{S}$ is the solvent molar mass, $a$ is the solvent activity, $P_{V S 0}$ is the saturated solvent vapor pressure, $\varphi_{S}$ is the solvent volume fraction in the liquid phase at the interface and $R$ is the ideal gas constant. In polymer solutions the activity is close to one for solvent volume fraction greater than about 0.4 [18], so that $\Phi_{e v}$ can be assumed independent of the solvent concentration at the beginning of the drying $(a \simeq 1)$.

Moreover, given the small amplitude of temperature variations observed in the experiments (a few degrees), it is possible to use a first order development of $\Phi_{e v}$, i.e.

$\Phi_{e v}(T(x, e, t)) \simeq \Phi_{e v}\left(T_{0}\right)+\left.\frac{\partial \Phi_{e v}}{\partial T}\right|_{T_{0}}\left(T(x, e, t)-T_{0}\right)$

The thermal boundary condition at the free surface can then be approximated by the following expression

$$
-k\left(\frac{\partial T}{\partial y}\right)_{y=e}=H_{t h}\left(T(x, e, t)-T_{0}\right)+L \Phi_{e v}\left(T_{0}\right)
$$

with $H_{t h}=h_{t h}+\left.L \frac{\partial \Phi_{e v}}{\partial T}\right|_{T_{0}}$

A shear stress boundary condition is imposed at the free surface, given by the 
balance of surface tension forces with the viscous stresses in the fluid.

$$
\mu\left(\frac{\partial v_{x}}{\partial y}\right)_{y=e}=\frac{d \gamma}{d T}\left(\frac{\partial T}{\partial x}\right)_{y=e}
$$

where $\gamma$, the surface tension, is a linearly decreasing function of temperature.

The other boundary condition at the interface concerns the vertical component of the velocity, $v_{y}$. Assuming a planar surface, and assuming that the spatial variations of the evaporation flux are much smaller than the flux itself, it can be shown [12] that, in the limit of $P e_{i n t} \ll 1$, the boundary condition reduces to:

$$
v_{y}=0 \quad \text { at } y=e .
$$

According to experimental results, the following values have been used: $H_{t h}=$ $28 W \cdot m^{-2} \cdot K^{-1}, L=396 \mathrm{~kJ} \cdot \mathrm{kg}^{-1}, \Phi_{e v}\left(T_{0}\right)=3.37 \times 10^{-4} \mathrm{~kg} \cdot \mathrm{m}^{-2} \cdot \mathrm{s}^{-1}, d \gamma / d T=$ $-0.119 \times 10^{-3} N \cdot m^{-1} \cdot K^{-1}$.

\subsection{Non-dimensional equations}

The non-dimensional form of the equations results from scaling the lengths by the thickness of the fluid $e$, the velocity $\vec{v}$ by the diffusion velocity $\alpha / e$, and time $t$ and pressure $p$ respectively by $e^{2} / \alpha$ and $\rho \alpha^{2} / e^{2}$. The temperature scale is $\theta=\frac{T-T_{0}}{\Delta T}$ where $\Delta T$ is the difference between the initial temperature $T_{0}$ and the steady temperature obtained at the end of the transient regime, when the temperature is uniform in the solution. From equation 5 we have $\Delta T=\frac{L \Phi_{e v}\left(T_{0}\right)}{H_{t h}}$, that is $\Delta T=4.8 \mathrm{~K}$ for the experimental configuration. Let us note that this temperature scale is different from the one used in classical stability analysis where the basic state is characterized by a constant temperature gradient in the fluid.

The dimensionless form of equations (1a-1c) is:

$$
\begin{gathered}
\vec{\nabla} \cdot \vec{v}=0 \\
\frac{\partial \vec{v}}{\partial t}+(\vec{v} \cdot \vec{\nabla}) \vec{v}=-\vec{\nabla} p+\operatorname{RaPr} \theta \vec{e}_{y}+\operatorname{Pr} \Delta \vec{v} \\
\frac{\partial \theta}{\partial t}+(\vec{v} \cdot \vec{\nabla}) \theta=\Delta \theta
\end{gathered}
$$

where $\operatorname{Pr}=\nu / \alpha$ is the Prandtl number, $R a=\beta_{T} g \Delta T e^{3} /(\nu \alpha)$ is the Rayleigh number. 
The dimensionless form of thermal (Eq. 5) and dynamic (Eq. 6 and 7) boundary conditions at the free surface are :

$$
\begin{gathered}
-\left(\frac{\partial \theta}{\partial y}\right)_{y=1}=B i(\theta(x, y=1, t)+1) \text { with } B i=\frac{H_{t h} e}{k} \\
\left(\frac{\partial v_{x}}{\partial y}\right)_{y=1}=-M a\left(\frac{\partial \theta}{\partial x}\right)_{y=1} \text { with } M a=-\frac{e \Delta T}{\mu \alpha}\left(\frac{\partial \gamma}{\partial T}\right) \\
v_{y}=0 \quad \text { at } y=e .
\end{gathered}
$$

with $M a$ the Marangoni number and $B i$ a modified Biot number that takes into account the evaporative flux.

The flow and thermal behaviour in the solution are governed by four nondimensional parameters $(B i, M a, P r$ and $R a)$, that depend on the two control parameters used in the experiments, the initial thickness and viscosity [13].

\subsection{Numerical method}

Computations are carried out with a collocated finite volume scheme with a second order space and time discretization [19]. The discrete scheme is fully coupled. The set of the discrete balance equations is solved by an under-relaxed Newton's method with the iterative linear solver BICGSTAB, preconditioned by an incomplete LU factorization. The chosen mesh is a standard regular mesh $L * e=(800 * 40)$ and the time step is equal to $\Delta t=10^{-3}$. Results obtained with finer grids or time steps did not show any noticeable changes in the results.

\section{Transient convection}

\subsection{Typical temperature and velocity fields}

Fig.2 gives a typical example of the time evolution of the non dimensional temperature difference between the bottom and the free surface of the solution for a configuration where convection is observed (parameters of the test case are given in table 1, initial velocity disturbance amplitude $r=4$ ). As a comparison, the temperature evolution obtained for a pure diffusive problem is also drawn (dashed line obtained with $M a=0$ and $R a=0$ ). Several domains can be observed. At the beginning, heat transfer is dominated by the diffusion and the two curves are superposed (domain I). Then convection 
starts and it is characterized first by strong and rapid changes of the temperature field (domain II). This regime is followed by a quasi-steady regime with a slow decrease of the temperature difference between the bottom and the free surface (domain III). At last for large times the two curves corresponding to pure diffusive or convective regimes go to zero. Indeed the temperature in the fluid is uniform at the end of the thermal transient regime (not represented in Fig.2).

The stream lines and the temperature on the free surface are given in Fig.3 for three dimensionless times $\mathrm{t}=0.5, \mathrm{t}=0.6$ and $\mathrm{t}=0.65$. We can observe the vanishing of the central cell between $t=0.5$ and $t=0.65$ that corresponds to the second peak that can be observed on the temperature evolution in Fig.2. It is followed by the quasi-steady regime, with a constant number of cells.

The objective of this paper is to analyze the three regimes, as a function of the four non dimensional parameters that characterize the problem, $B i$, $M a, \operatorname{Pr}$ and $R a$ numbers. The transition from domain I to domain II, i.e. the onset of convection is analyzed in section 4 . Section 5 is dedicated to the characterization of the quasi-steady regime, using scaling laws to get an estimation of the temperature and velocity in this regime.

To characterize the presence of observable convection, a criterium based on the thermal Peclet number was chosen. The thermal Peclet number $P e=e v / \alpha$ compares the relative importance of advection and diffusion. The velocity $v$ used in the estimation of the Peclet number is the maximal value of the velocity norm. Then convection will be considered significant if, when the system is submitted to an initial velocity perturbation, there is a time $t$ where the perturbation is significantly amplified, i.e. such as $d P e(t) / d t>0$ and $P e(t)>1$.

\subsection{Effect of the initial disturbance amplitude}

As the problem is sensitive to initial conditions, we have first performed a preliminary study to analyze the influence of the amplitude of the initial disturbance on the time evolution of the Peclet number. The parameters used for this test are given in Tab.1.

Fig.4a shows the temporal evolution of the Peclet number in log scale. At the beginning a linear regime can be observed with a decreasing of the initial perturbation followed by its amplification. But at large $t$, in the non linear regime, $P e$ becomes quasi independent of the initial perturbation. If we consider the chosen criterium $(P e>1)$, all the tests lead to the same conclusion, i.e. convection is observed; only the time delay depends on the amplitude of the disturbance. The corresponding time is about $t=0.1$ for $r=4$ and $t=0.03$ 
for $r=400$. Fig.4b shows the Peclet number normalized by its initial value, so that the transition between the linear and non linear regime can be clearly observed.

Since the general trends observed in the three regimes and the occurrence of convection do not depend strongly on the perturbation amplitude (at least in a large domain), we use $r=4$ in the following. A more detailed stochastic analysis of the influence of the initial perturbation structure has been performed [17] and gives the same conclusions: changing the initial perturbation induces only small changes in the pattern wavelength, in the temperature or in the Peclet variations. The threshold value corresponding to the onset of convection is therefore little sensitive to the initial perturbation. This assumption is confirmed in section 4.3 by the good agreement between the thresholds obtained with the non-linear simulations and the linear stability analysis. However the threshold must not be understood as a precise delimitation between stable and unstable domains but rather as a transition region (a factor of about two is obtained for different initial perturbations). This "blurring" effect is inherent to the transient character of the problem under study [12].

\section{Stability}

\subsection{Influence of the dynamic viscosity}

The viscosity of solutions understudy being very sensitive to the initial polymer concentration, we first study the influence of the dynamic viscosity $\mu$ on the Peclet transient behavior. Both buoyancy and surface tension driving forces are taken into account (Rayleigh-Bénard-Marangoni configuration) and results are given in Fig.5 for $e=1 \mathrm{~mm}$ and for different values of the dynamic viscosity $\mu$.

For the largest viscosities, the Peclet number is a monotone decreasing functions and the initial perturbation dies down. For smaller viscosities, there is an amplification of the initial perturbation after some times. However this amplification may be very weak and for viscosity larger than $5 \mathrm{mPa}$.s the Peclet number is always smaller than one, so that this configuration is "stable" according to the criterium defined in section 3.1. The critical viscosity corresponding to the onset of convection lies between $4 \mathrm{mPa}$.s and $5 \mathrm{mPa}$.s for this thickness. In the next section, the same study was performed for other thicknesses in order to compare simulations and experimental data. 


\subsection{Comparison of simulations and experiments}

Comparison is made with experimental points in the range of $\mathrm{Bi}, \mathrm{Ma}, \mathrm{Pr}$ and $R a$ covered by the variation of the two experimental control parameters (initial thickness and viscosity), that is:

$0.06 \leq B i \leq 5 ; \quad 6.6 \leq \operatorname{Pr} \leq 2.510^{4} ; \quad 20 \leq M a \leq 1.210^{5} ; \quad 1.3 \leq R a \leq$ $1.410^{6}$

Numerical simulations have been performed for $1 \mathrm{~mm}<e<30 \mathrm{~mm}$. For each thickness, we look for the maximum viscosity such as the convection criterium is fulfilled. This systematic study leads to the construction of the transition boundary between the "stable" and "unstable" domain in the plane thicknessviscosity, according to the criterium previously defined. Numerical results and experimental points are given in Fig .6. When the whole Rayleigh-BénardMarangoni problem (RBM) is considered (blue line), a change in the frontier slope is observed around $e=8 \mathrm{~mm}$. As already known for RBM configuration [1] this corresponds to the transition between the domain dominated by Bénard-Marangoni (BM) instabilities (small thicknesses) and Rayleigh-Bénard (RB) instabilities (large thicknesses). To illustrate this point, the same simulations were performed canceling the buoyancy term or the surface tension term in the Navier Stokes equation. The corresponding thresholds are compared in Fig.6. For thicknesses smaller than a few millimeters the curves with or without buoyancy are similar and Marangoni effects are dominant. The reverse is observed for thicknesses larger than $8 \mathrm{~mm}$, where buoyancy effects become dominant. This confirms experimental results where two different morphologies of convective patterns had been observed for small and large thicknesses respectively (transition from cells to a mixture of cells and rolls, cf. [13]). Agreement between experimental observations and simulated thresholds is good, except for some experiments located in the "stable" domain and exhibiting convective patterns. This point is discussed in the next section.

\subsection{Comparison with linear stability analysis}

The results obtained in this paper with direct non linear simulation are compared to a linear stability analysis otherwise performed and detailed in [12]. For this transient problem, a specific method was used, based on the nonnormal approach. First, as usually done, linear perturbation equations were derived. Two amplification gains were defined. The first one $G_{V}(t)$ is based on the kinetic energy of velocity perturbations and the second one $G_{T}(t)$ is a quadratic term based on temperature perturbation. Then for each wavenumber $k$ (spatial development of the perturbation in the infinite horizontal direction) and each time $t$ an optimization problem was solved in order to get the ver- 
tical profile $(0<y<e)$ of the initial optimal perturbation which leads to the maximization of $G_{V}(t, k)$ (velocity perturbation) or $G_{T}(t, k)$ (temperature perturbation). For a given set of non dimensional parameters ( $R a, M a, P r$ and $B i$ numbers) we then define $G_{V}^{*}=\operatorname{Max}_{t, k} G_{V}$, the larger amplification for any time and wavenumber when the initial perturbation is imposed on the velocity, and $G_{T}^{*}=\operatorname{Max}_{t, k} G_{T}$ that is the larger amplification for any time and wavenumber when the initial perturbation is imposed on the temperature. $G_{V}$ and $G_{T}$ are normalized with the initial values of the kinetic energy or temperature norm, so that $G^{*}<1$ means that the initial perturbation is never amplified [12].

The different criteria are compared in Fig.7. As can be seen, the boundaries obtained with velocity perturbations either with non linear simulations (criterium "Pe $=1$ ") or with linear analysis (" $G_{V}^{*}=1$ ") are very close. Perturbations on the temperature field (with criterium " $G_{T}^{*}=1$ ") give thresholds above the other ones, but, as previously said, the thresholds must rather be seen as a transition region, and all the criteria lead to the same order of magnitude.

At last, the pattern wavelengths obtained from simulations (quasi-steady regime) and experiments have also been compared and are in good agreement, as shown in Fig.8. As already obtained in RBM studies [20], no change is observed at the transition from surface tension driven flow to buoyancy driven flow, that occurs for a thickness around $1 \mathrm{~cm}$.

\subsection{Critical Marangoni and Rayleigh numbers}

Beyond the experiments a more general analysis of the influence of the Biot and Prandtl numbers on the stability thresholds have been performed. In the case of pure Bénard-Marangoni flow, the critical Marangoni number is shown to depend very few on the Prandtl number and to depend non monotonically on the Biot number, with a minimum value around $B i=2$ (Fig.9). Comparison with results obtained with the linear analysis and criterium $G_{V}^{*}=1$ [12] is made in Fig.9. The thresholds obtained with the two approaches are very close and two asymptotic power laws can be defined: "Bi $M a \simeq$ constant" and "Bi$\simeq$ constant $M a$ " for low and large values of Biot numbers respectively. Similar results (not presented here) have been obtained with the RB configuration, i.e. linear and non linear results are very close. 


\section{Scaling laws in the quasi-steady regime}

This last section is devoted to the analysis of the quasi-steady regime in the Bénard-Marangoni case $(R a=0)$. For such conditions, we aim to obtain the scaling laws that give the order of magnitude of the temperature variations and of the velocity inside the liquid layer, as a function of the three nondimensional parameters $M a, B i$ and $P r$. This objective is reached by solving equations $8 \mathrm{a}$ to 11 in terms of order of magnitude (scaling analysis, cf. for instance ref. [21] for an example of scaling derivation applied to a transient free convection problem). The derivation is made possible by use of symplifying assumptions listed below.

First, the existence of the quasi steady regime is assumed a priori. The obtained results are then restricted to the domain where convection is expected, i.e. the domain above the two asymptotical lines defined in Fig.9 (cf. [12] for a detailed analysis of the asymptotical values). The following assumptions are made:

H1 we assume the existence of an hydrodynamic boundary layer (resp. thermal boundary layer) of thickness $\delta_{H}\left(\operatorname{resp} . \delta_{T}\right)$ below the free surface.

$\mathrm{H} 2$ time derivative terms in equations (1b) and (1c) are neglected (quasisteady regime assumption).

H3 the vertical and horizontal temperature variations across a convective cell are of same order of magnitude, denoted $\Delta \theta$ (compare for instance Figs .2 and $.3)$.

H4 the wavelength of convective structures is of the order of the thickness (cf. Fig.8), so that the order of magnitude of the characteristic length scale in the $x$-direction is 1 .

H5 the analysis is restricted to fluid with $\operatorname{Pr} \gtrsim 1^{1}$.

In addition, the order of magnitude of the velocity component parallel to the free surface in the hydrodynamic layer is denoted $v_{x}$, while the order of magnitude of the vertical velocity component is denoted $v_{y}$.

\section{1 equations}

In the following, the equations of section 2.3 are written in terms of order of magnitude.

* Thanks to H1, H3 and H4, the shear stress boundary condition (equation

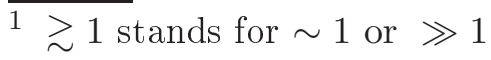


(10)) reads :

$$
\frac{v_{x}}{\delta_{H}} \sim M a \Delta \theta
$$

* The mass conservation (equation (8a)), with assumptions H1 and H4 reads:

$$
v_{x} \sim \frac{v_{y}}{\delta_{H}}
$$

* Momentum conservation.

Following a classical derivation, the pressure gradient term can be eliminated from equations $(8 \mathrm{~b})$ :

$\frac{1}{\operatorname{Pr}}\left[\frac{\partial}{\partial x}\left(\frac{\partial v_{y}}{\partial t}+v_{x} \frac{\partial v_{y}}{\partial x}+v_{y} \frac{\partial v_{y}}{\partial y}\right)-\frac{\partial}{\partial y}\left(\frac{\partial v_{x}}{\partial t}+v_{x} \frac{\partial v_{x}}{\partial x}+v_{y} \frac{\partial v_{x}}{\partial y}\right)\right]=\frac{\partial}{\partial x}\left(\frac{\partial^{2} v_{y}}{\partial x^{2}}+\frac{\partial^{2} v_{y}}{\partial y^{2}}\right)-\frac{\partial}{\partial y}\left(\frac{\partial^{2} v_{x}}{\partial x^{2}}+\frac{\partial^{2} v_{x}}{\partial y^{2}}\right)$

This relation states a balance between inertia (left-hand side) and friction (right-hand side). The times derivatives $\partial_{t} v_{x}$ and $\partial_{t} v_{y}$ can be dropped out thanks to the assumption of quasi-steady regime (H2). To go further, we need to distinguish two cases : $\delta_{H} \ll 1$ or $\delta_{H} \sim 1$. According to equation (13), the condition $\delta_{H} \ll 1$ implies $v_{x} \gg v_{y}$. In that case, it is easy to show that, in terms of order of magnitude, equation (14) reads $v_{x}^{2} /\left(\operatorname{Pr} \delta_{H}\right) \sim v_{x} / \delta_{H}^{3}$, which leads to:

$$
\delta_{H} \sim \sqrt{\frac{P r}{v_{x}}}
$$

This equation is valid for $\sqrt{\operatorname{Pr} / v_{x}} \ll 1$ only, since it was established by assuming $\delta_{H} \ll 1$. In the opposite case such that $\sqrt{\operatorname{Pr} / v_{x}} \gtrsim 1$, the hydrodynamic boundary layer thickness saturates at $\delta_{H} \sim 1$, and inertia is no more involved in the problem.

\section{* Energy conservation.}

Denoting $v_{y T}$ the order of magnitude of the vertical velocity component inside the thermal boundary layer, the equation (8c) is a balance between four terms, due to advection and diffusion. With assumptions H3, we get the following terms $\left(v_{x} \Delta \theta ; v_{y T} \Delta \theta / \delta_{T} ; \Delta \theta ; \Delta \theta / \delta_{T}^{2}\right)$. Once again two cases must be considered, according to the value of $\delta_{T}$. In the case $\delta_{T} \ll 1$, one can easily show that

$$
\delta_{T} \sim \frac{1}{\sqrt{v_{x}}}
$$

This relation is valid if $1 / \sqrt{v_{x}} \ll 1$ only. In that case, the thermal boundary layer thickness results from a balance between advection and diffusion. In the opposite case $1 / \sqrt{v}_{x} \gtrsim 1$, it saturates at $\delta_{T} \sim 1$, and another regime occurs where advection is negligible compared to diffusion.

* Thermal boundary condition.

The equation (9) states a balance between three terms, characterizing respec- 
tively the diffusion in the liquid, the heat supplied by convection from the gaz phase, and the cooling due to evaporation :

$$
\frac{\Delta \theta}{\delta_{T}} ; B i \Delta \theta ; B i
$$

Two regimes can be considered, depending on which terms dominate the heat balance at the interface. If the condition $\Delta \theta \ll 1$ is satisfied, the heat flux from convection in the gas phase is negligible, and the energy needed by evaporation is balanced by the diffusion in the liquid. The equation (17) hence reads:

$$
\Delta \theta \sim B i \delta_{T}
$$

The equation (18) is valid if $B i \delta_{T} \ll 1$ only. Conversely, another regime occur if $B i \delta_{T} \gtrsim 1$, characterized by the temperature variation saturating at $\Delta \theta \sim 1$. In that case the energy is supplied by convection in the gas phase, since the terms $B i \Delta \theta$ and $B i$ dominate in equation (17).

\subsection{Synthesis of the different regimes}

The previous section shows that we must consider a combination of the following cases:

$$
\begin{array}{cccc}
\delta_{H} \ll 1 & \text { or } & \delta_{\mathrm{H}} \sim 1 \\
\delta_{T} \ll 1 & \text { or } & \delta_{\mathrm{T}} & \sim 1 \\
\Delta \theta \ll 1 & \text { or } & \Delta \theta & \sim 1
\end{array}
$$

We see from equations (15) and (16) that only cases where $\delta_{H} \gtrsim \delta_{T}$ are to be taken into account since the analysis is restricted to fluid with $\operatorname{Pr} \gtrsim 1$ (H5 assumption). Moreover, only unstable configurations are considered and, in the $M a / B i$ plane, the domain is restricted to the points above the two asymptotical curves of the critical Marangoni obtained from the complete stability analysis, i.e. $B i \sim M a^{-1}$ for small Biot numbers and $B i \sim M a$ for large Biot numbers (cf. Fig.9). Taking into account these remarks, we obtain the five domains listed in table .2 and Fig.10. Correlations and boundaries between the different domains are deduced from the previous equations. Their derivation is detailed in the annex.

At last Fig.11 shows an illustration of the scaling laws in the domain B, deduced from simulations performed for configurations close to the experimental conditions. As can be seen numerical simulations are in good agreement with scaling laws. 


\section{Conclusion}

In this paper a numerical analysis of a thermal transient problem induced by solvent evaporation in an initially isothermal fluid is considered. The thermal behavior shows the succession of three steps: the first one, before the onset of convection, is purely diffusive, the second step corresponds to the beginning of convection and important variations in temperature and velocity fields are observed, and the last one is characterized by a very slow evolution of the system towards the final isothermal state. The problem is described by four non-dimensional parameters, $B i, M a, \operatorname{Pr}$ and $R a$. Our approach takes into account explicitly the transient character of the problem. A criterium based on the Peclet number is used to characterize the onset of convection. It is important to notice that the threshold must not be understood as a precise delimitation between stable and unstable domains but rather as a transition domain, since the obtained threshold values depend slightly on the initial perturbation. The complete problem (i.e. convection induced by thermocapillarity and buoyancy) or the pure Rayleigh-Bénard and pure Bénard-Marangoni configuration is considered. It was found that the obtained thresholds do not depend on the value of the $\operatorname{Pr}$ number significantly.

Results of the non linear simulations are compared with a linear transient stability analysis based on a non normal approach [12]. Stability thresholds obtained with the two approaches are very close, which validates the linear approach for the determination of the onset of convection. Results also well compare with experimental observations obtained from experiments of PIB/Toluene solutions drying [13], for stability thresholds and convective structures wavelengths. This validates the simplifying assumptions made to simulate the beginning of the drying where only thermal phenomena have been taken into account.

At last a scaling analysis is developed for the quasi-steady regime in the BM configuration. Correlations are derived to get the order of magnitude of the temperature and velocity as a function of $B i, M a$ and $\operatorname{Pr}$ numbers. Further developments would imply to get a better description of the coupling between the liquid and the gas (two layers model) and to allow deformations of the interface. Other development concerns the second part of the drying process when solutal convection takes place. This problem is more complex since the assumption of constant physical properties and fixed interface are no more valid.

\section{Acknowledgment}

The authors thank M. Rossi from "Institut Jean Le Rond d'Alembert, CNRSUPMC, France" and T.Boeck from "Technische Universität Ilmenau, Ger- 
many" for helpful discussions. This work was supported by the " Fédération TMC (Ministère de l'Enseignement supérieur et de la Recherche, France) " 
Annex: Scaling analysis: derivation of correlations and boundaries

Derivation of correlations is detailed for domain B only, since the method is the same for all the domains. From equation 13 it can be seen that $v_{x} \gtrsim v_{y}$, so that the order of magnitude of the Peclet number is given by $v_{x}$

Domain $\mathrm{B}$ is characterized by $\Delta \theta \ll 1, \delta_{T} \ll 1$ and $\delta_{H} \sim 1$. It is then described by equations $12,13,16$ and 18 . Correlations given in table .2 follows easily from this set of equations with the condition $\delta_{H} \sim 1$.

The boundaries between the domain $\mathrm{B}$ and adjacent domains are obtained in the following way: Equation 16 and condition $\delta_{T} \ll 1$ gives $v_{x} \gg 1$ in domain $\mathrm{B}$. Then the boundary between domain A (no convection, i.e. very small velocities) and $\mathrm{B}$ is obtained writing $v_{x} \sim 1$, that is $P e \sim 1$ and then $M a B i \sim 1$. The domains $\mathrm{B}$ and $\mathrm{C}$ differ by the temperature difference $\Delta \theta$ which is close to one in domain $\mathrm{C}$. The boundary between the two domains is then obtained writing $\Delta \theta \sim 1$ in the correlation $\Delta \theta \sim M a^{-1 / 3} B i^{2 / 3}$ of domain $\mathrm{B}$. In the same way, the boundary between domain $\mathrm{B}$ and $\mathrm{E}$ is obtained writing $\delta_{H} \sim 1$ in the correlation $\delta_{H} \sim(M a B i)^{-1 / 4} \mathrm{Pr}^{3 / 8}$ of domain E. The other boundary equations follow from the same kind of arguments. However, let us note that the boundary between domains $\mathrm{C}$ and $\mathrm{A}$ deduced from scaling laws is $\mathrm{Ma} \sim 1$, while asymptotes deduced from stability analysis reads $\mathrm{Bi} \sim \mathrm{Ma}$ (in terms of order of magnitude). As previously said, scaling laws have been derived assuming the existence of the quasi-steady regime. The differences between the two boundaries mean that the quasi-steady regime is not reached for points below $\mathrm{Bi} \sim \mathrm{Ma}$.

\section{References}

[1] J. Pearson, On convection cells induced by surface tension, J. Fluid Mech. 4 (1958) 489-500.

[2] F. Busse, Non-linear properties of thermal convection, Rep. Prog. Phys. 41 (1978) 1930-1967.

[3] J. Reichenbach, H. Linde, Linear perturbation analysis of surface-tension-driven convection at a plane interface (Marangoni instability), J. of colloid and interface science 84 (1981) 433-443.

[4] D. Goussis, R. Kelly, On the thermocapillary instabilities in a liquid layer heated from below, Int J. Heat Mass Transfer 33 (1990) 2237-2245.

[5] P. Colinet, J. C. Legros, M. G. Velarde, Nonlinear Dynamics of Surface-TensionDriven Instabilities, Wiley-VCH, 2001.

[6] P. Manneville, Dynamics of Spatio-Temporal Cellular Structures, Henri Bénard Centenary Review, Springer tracts in Modern Physics, 2006. 
[7] D. Merkt, M. Bestehorn, Bénard-Marangoni convection in a strongly evaporating fluid, Physica D 185 (2003) 196-208.

[8] P. Colinet, L. Joannes, C. S. Iorio, B. Haute, M. Bestehorn, G. Lebon, J. C. Legros, Interfacial turbulence in evaporating liquids: Theory and preliminary results of the itel-master 9 sounding rocket experiment, Advances in Space Research 32 (2) (2003) 119-127.

[9] O. Ozen, R. Narayanan, The physics of evaporative and convective instabilities in bilayer systems: Linear theory, Phys. Fluids 16 (2004) 4644-4652.

[10] C. Moussy, G. Lebon, J. Margerit, Influence of evaporation on BénardMarangoni instability in a liquid-gas bilayer with a deformable interface, Eur. Phys. J. B 40 (2004) 327-335.

[11] S. Medale, P. Cerisier, Numerical simulation of Bénard-Marangoni convection in small aspect ratio containers, Num. Heat Transf. A 42 (2002) 55-72.

[12] F. Doumenc, T. Boeck, B. Guerrier, M. Rossi, Rayleigh-Bénard-Marangoni convection due to evaporation : a linear non-normal stability analysis, submitted to JFM.

[13] G. Toussaint, H. Bodiguel, F. Doumenc, B. Guerrier, C. Allain, Experimental characterization of buoyancy- and surface tension-driven convection during the drying of a polymer solution, Int. J. Heat Mass Transfer 51 (2008) 4228-4237.

[14] M. Doi, S. Edwards, The theory of polymer dynamics, Oxford University Press, 1988.

[15] S. H. Davis, Thermocapillary instabilities, Annu. Rev. Fluid Mech. 19 (1987) 403-435.

[16] Y. Gorand, F. Doumenc, B. Guerrier, C. Allain, Instabilités de plissement lors du séchage de films polymères plans, Rhéologie 3 (2003) 22-29.

[17] E. Chenier, C. Desceliers, B. Trouette, Sensibilité à la condition initiale de la convection naturelle de Bénard-Marangoni en régime transitoire, in: Proceedings SFT Conference, 2009.

[18] F. Doumenc, B. Guerrier, C. Allain, Mutual diffusion coefficient and vaporliquid equilibrium data for the system pib/toluene, Journal of Chemical and Engineering Data 50 (2005) 983-988.

[19] E. Chénier, R. Eymard, O. Touazi, Numerical results using a colocated finite volume scheme on unstructured grids for incompressible flows, Numerical Heat Transfer, part. B (2006) 1-18.

[20] D. Nield, Surface tension and buoyancy effects in cellular convection, J. Fluid Mech. 19 (1964) 341-352.

[21] A. Bejan, Convection Heat Transfer, Wiley Interscience, 1984. 


\section{List of Figures}

$.1 \quad$ Viscosity, experimental points and interpolation curve.

.2 Time evolution of the temperature difference between the bottom and top of the layer for the convective model. $\theta(x=A / 2, y=0, t)-\theta(x=A / 2, y=1, t)$ (grey continuous line $),<\theta(y=0, t)>-<\theta(y=1, t)>$ (black continuous line), pure diffusive model (dashed dotted line)- test case of table 1.

.3 Stream lines and temperature at the free surface for $\mathrm{t}=0.5$, $\mathrm{t}=0.6$ and $\mathrm{t}=0.65$ - test case of table 1 .

.4 Evolution of (a) Peclet number Pe(t) and (b) $P e(t) / P e(t=0)$ for three values of the initial disturbance: $r=4,40,400$ - test case of table 1 .

.5 Péclet number for $e=1 \mathrm{~mm}$ and various dynamic viscosities.

.6 Comparison of RBM, BM and RB configurations. Symbols refer to experimental results: no convection (red squares), convective patterns (blue stars). Lines refer to simulations: thresholds obtained with RBM configuration (blue continuous line), BM configuration (red dashed line) and RB configuration (green dotted line).

.7 Comparison of theory and experiments. Experimental results: same symbols than in Fig.6. Simulation thresholds obtained with the following criteria: linear analysis and $G_{T}^{*}=1$ (red dotted line), linear analysis and $G_{V}^{*}=1$ (red dashed line), non linear analysis and $P e=1$ (green continuous line).

.8 Wavelengths of the convective patterns, experimental points (blue triangles) and simulations (red cross).

.9 Comparison of critical Marangoni obtained with linear and non linear analysis. non linear analysis and $P r=1$ (red empty triangles), linear analysis and $P r=1$ (red full triangles), non linear analysis and $\operatorname{Pr}=1000$ (blue empty circles), linear analysis and $\operatorname{Pr}=1000$ (blue full circles). The dashed black lines are asymptotical lines with slope +1 and -1 .

.10 Quasi-steady regime in the BM configuration. Domains derived from the scaling analysis (the figure is drawn for $\operatorname{Pr}=100$ ). 
.11 Comparison of simulations and correlations in the B domain. (a) $P e$ versus BiMa, simulations (crosses) and straight line with slope $2 / 3$, (b) $P e$ versus $B i^{2} / M a$, simulations (stars) and straight line with slope $1 / 3$. 


\begin{tabular}{|c|c|c|c|c|c|}
\hline e[mm] & $\mu[\mathrm{mPa} . \mathrm{s}]$ & $B i$ & $\operatorname{Pr}$ & $M a$ & $R a$ \\
\hline 1 & 1 & 0.2 & 12 & 5850 & 451 \\
\hline
\end{tabular}

Table .1

Testcase parameters.

\begin{tabular}{|c|c|c|c|c|}
\hline domain & $\Delta \theta$ & $\delta_{T}$ & $\delta_{H}$ & correlations \\
\hline $\mathrm{A}$ & & & & no convection \\
\hline B & $\ll 1$ & $\ll 1$ & $\sim 1$ & $\begin{array}{c}\delta_{T} \sim(M a \cdot B i)^{-1 / 3} \\
\Delta \theta \sim M a^{-1 / 3} \cdot B i^{2 / 3}, P e \sim(M a . B i)^{2 / 3}\end{array}$ \\
\hline $\mathrm{C}$ & $\sim 1$ & $\ll 1$ & $\sim 1$ & $\begin{array}{c}\delta_{T} \sim M a^{-1 / 2} \\
P e \sim M a\end{array}$ \\
\hline $\mathrm{D}$ & $\sim 1$ & $\ll 1$ & $\ll 1$ & $\begin{array}{c}\delta_{T} \sim M a^{-1 / 3} \cdot \operatorname{Pr}^{-1 / 6}, \delta_{H} \sim M a^{-1 / 3} \cdot \operatorname{Pr}^{1 / 3} \\
P e \sim M a^{2 / 3} \cdot \operatorname{Pr}^{1 / 3}\end{array}$ \\
\hline $\mathrm{E}$ & $\ll 1$ & $\ll 1$ & $\ll 1$ & $\begin{array}{l}\delta_{T} \sim(M a \cdot B i)^{-1 / 4} \cdot \operatorname{Pr}^{-1 / 8}, \delta_{H} \sim(M a \cdot B i)^{-1 / 4} \cdot \operatorname{Pr}^{3 / 8} \\
\Delta \theta \sim M a^{-1 / 4} \cdot B i^{3 / 4} \cdot \operatorname{Pr}^{-1 / 8}, P e \sim(M a \cdot B i)^{1 / 2} \cdot \operatorname{Pr}^{1 / 4}\end{array}$ \\
\hline
\end{tabular}

Table .2

Quasi-steady regime in the BM configuration - Correlations.

Table .3

\begin{tabular}{|c|c|c|}
\hline domain & domain & boundary equation \\
\hline A & B & $B i \times M a \sim 1$ \\
\hline A & C & $B i \sim M a$ \\
\hline B & C & $B i^{2} \times M a^{-1} \sim 1$ \\
\hline B & E & $B i^{2 / 3} \times M a^{2 / 3} \sim P r$ \\
\hline C & D & $M a \sim P r$ \\
\hline D & E & $B i^{6} \times M a^{-2} \sim P r$ \\
\hline
\end{tabular}

Quasi-steady regime in the BM configuration - boundaries between the different domains. 


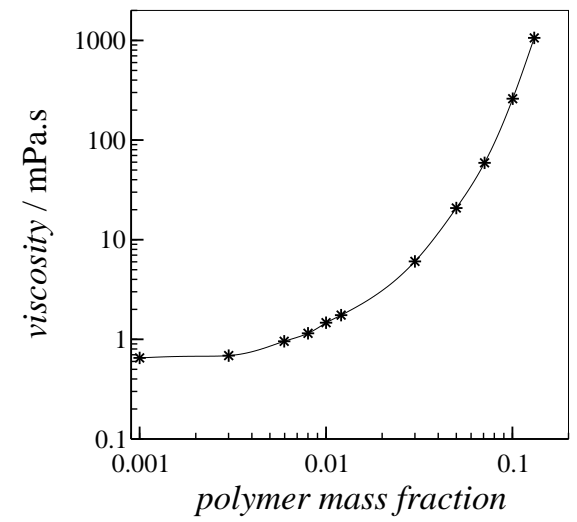

Fig. .1. Viscosity, experimental points and interpolation curve.

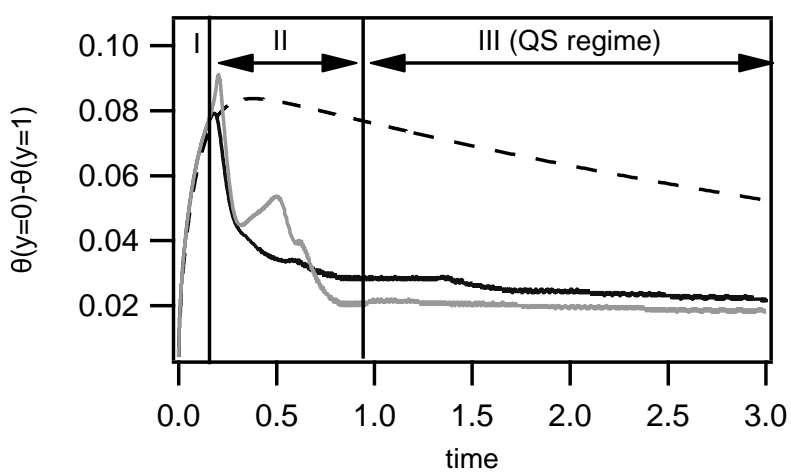

Fig. .2. Time evolution of the temperature difference between the bottom and top of the layer for the convective model. $\theta(x=A / 2, y=0, t)-\theta(x=A / 2, y=1, t)$ (grey continuous line), $<\theta(y=0, t)>-<\theta(y=1, t)>$ (black continuous line), pure diffusive model (dashed dotted line)- test case of table 1. 


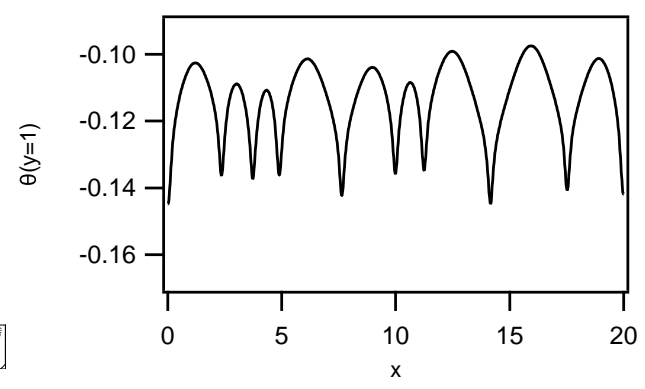

(a) $\mathrm{V}(\mathrm{x}, \mathrm{y}, \mathrm{t}=0.5)$

(b) $\theta(x, 1, t=0.5)$

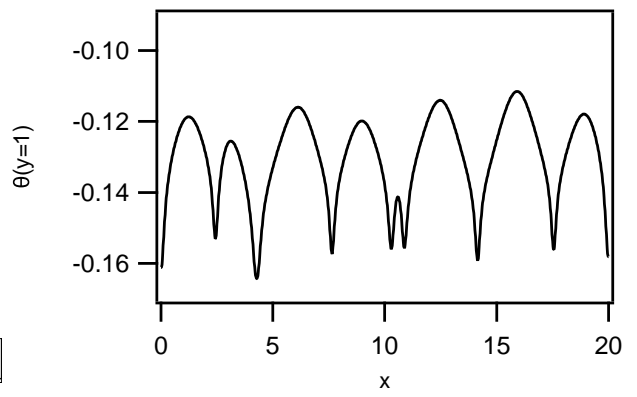

(c) $\mathrm{V}(\mathrm{x}, \mathrm{y}, \mathrm{t}=0.6)$

(d) $\theta(x, 1, t=0.6)$

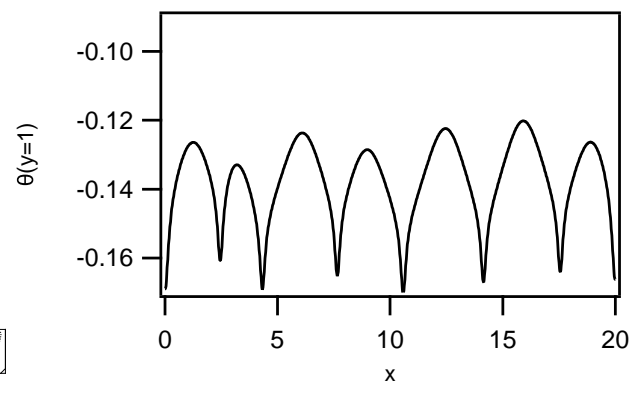

(e) $\mathrm{V}(\mathrm{x}, \mathrm{y}, \mathrm{t}=0.65)$

(f) $\theta(x, 1, t=0.65)$

Fig. .3. Stream lines and temperature at the free surface for $\mathrm{t}=0.5, \mathrm{t}=0.6$ and $\mathrm{t}=0.65$ - test case of table 1.

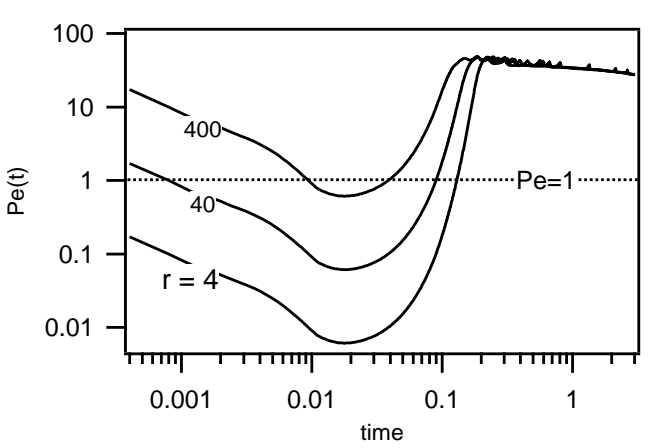

(a)

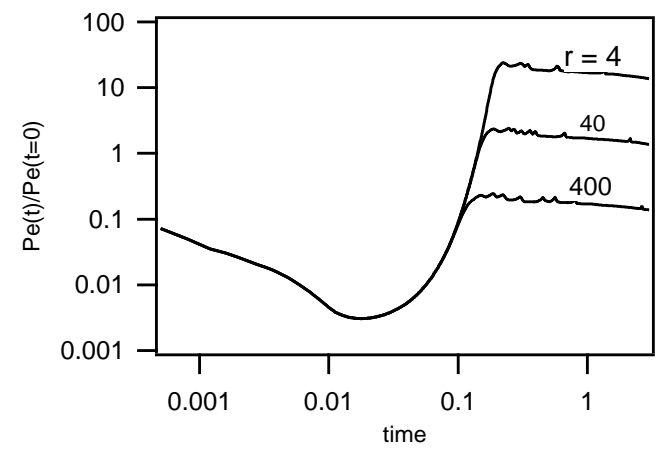

(b)

Fig. .4. Evolution of (a) Peclet number $\mathrm{Pe}(\mathrm{t})$ and (b) $\mathrm{Pe}(t) / \mathrm{Pe}(t=0)$ for three values of the initial disturbance: $r=4,40,400$ - test case of table 1 . 


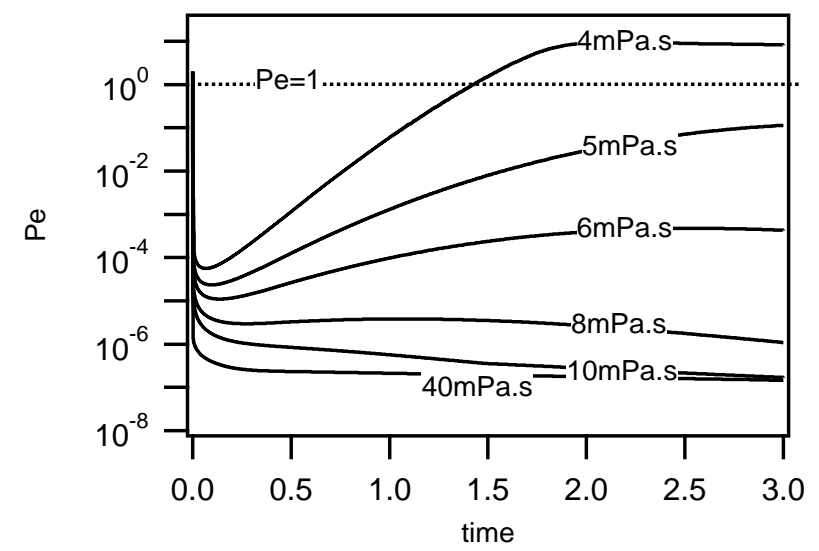

Fig. .5. Péclet number for $e=1 \mathrm{~mm}$ and various dynamic viscosities.

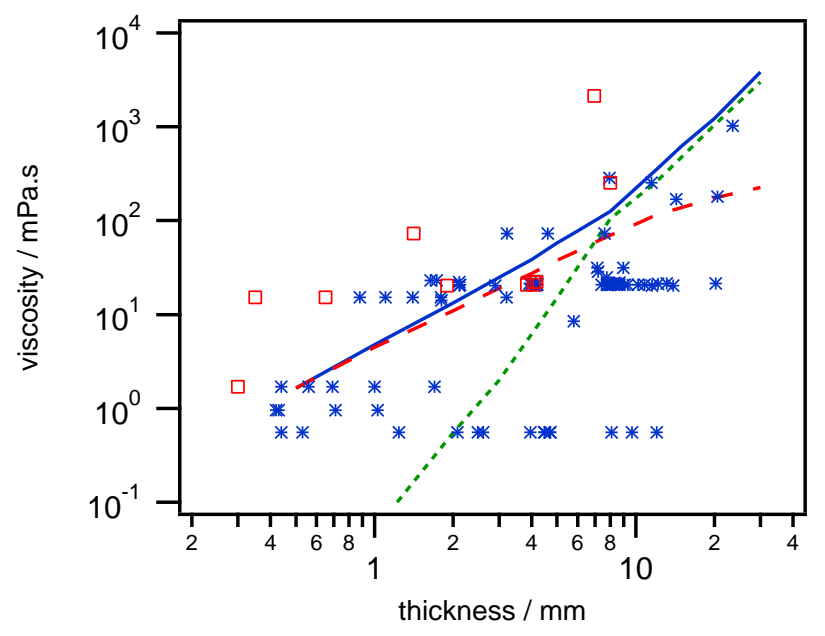

Fig. .6. Comparison of RBM, BM and RB configurations. Symbols refer to experimental results: no convection (red squares), convective patterns (blue stars). Lines refer to simulations: thresholds obtained with RBM configuration (blue continuous line), BM configuration (red dashed line) and RB configuration (green dotted line). 


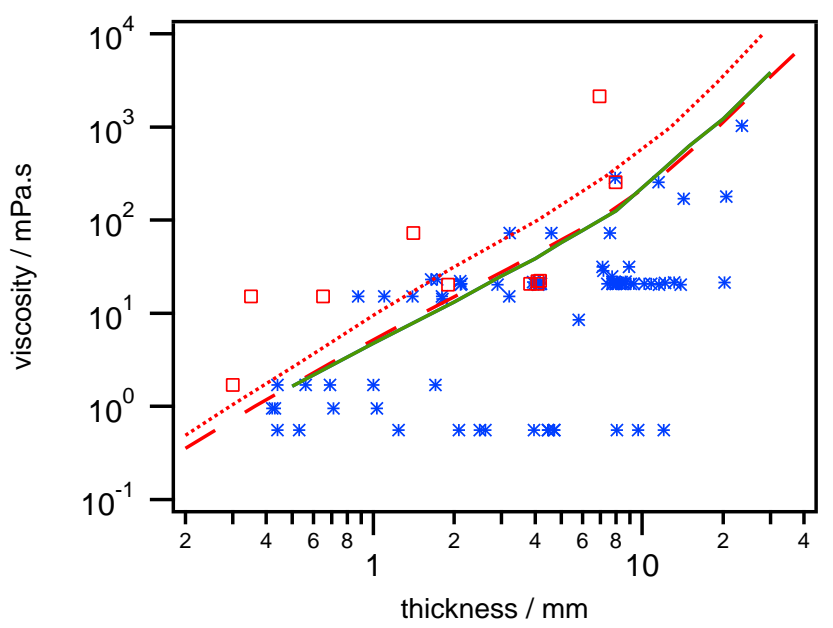

Fig. .7. Comparison of theory and experiments. Experimental results: same symbols than in Fig.6. Simulation thresholds obtained with the following criteria: linear analysis and $G_{T}^{*}=1$ (red dotted line), linear analysis and $G_{V}^{*}=1$ (red dashed line), non linear analysis and $P e=1$ (green continuous line).

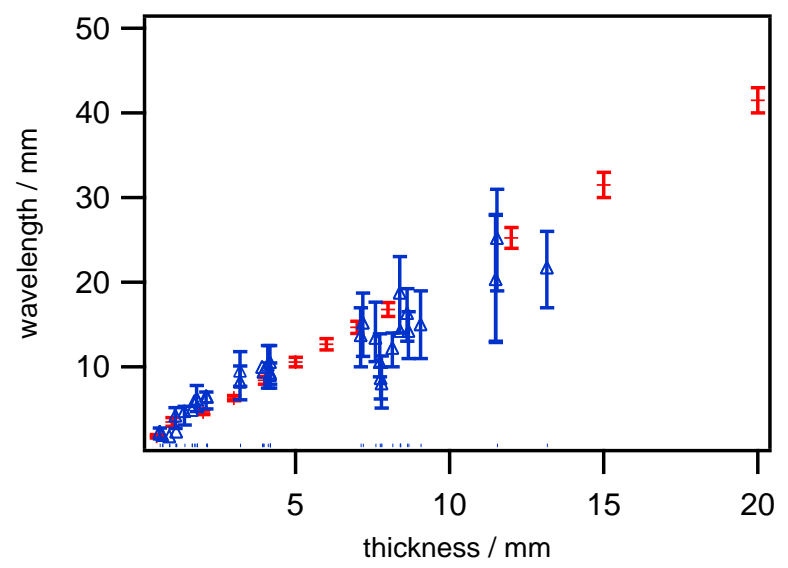

Fig. .8. Wavelengths of the convective patterns, experimental points (blue triangles) and simulations (red cross). 


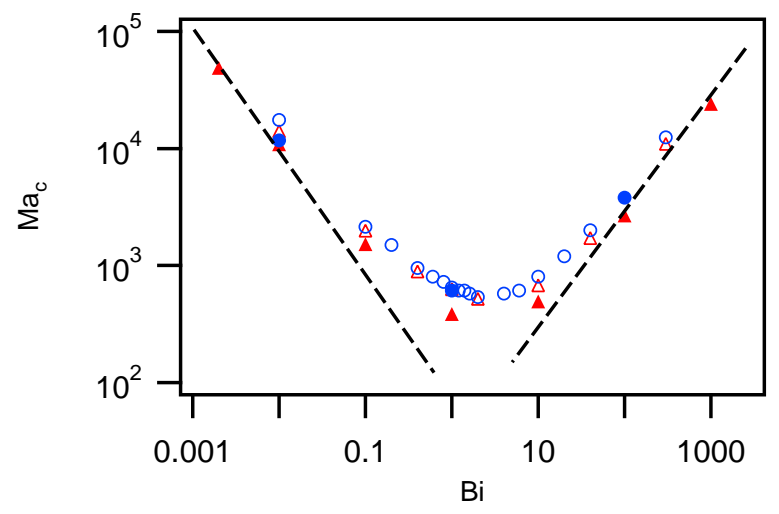

Fig. .9. Comparison of critical Marangoni obtained with linear and non linear analysis. non linear analysis and $\operatorname{Pr}=1$ (red empty triangles), linear analysis and $\operatorname{Pr}=1$ (red full triangles), non linear analysis and $P r=1000$ (blue empty circles), linear analysis and $\operatorname{Pr}=1000$ (blue full circles). The dashed black lines are asymptotical lines with slope +1 and -1 .

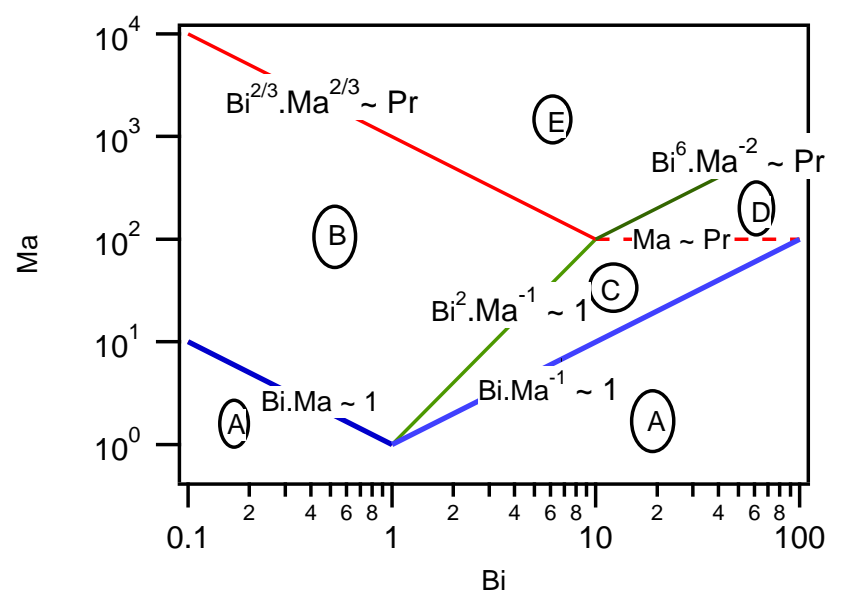

Fig. .10. Quasi-steady regime in the BM configuration. Domains derived from the scaling analysis (the figure is drawn for $\mathrm{Pr}=100$ ).

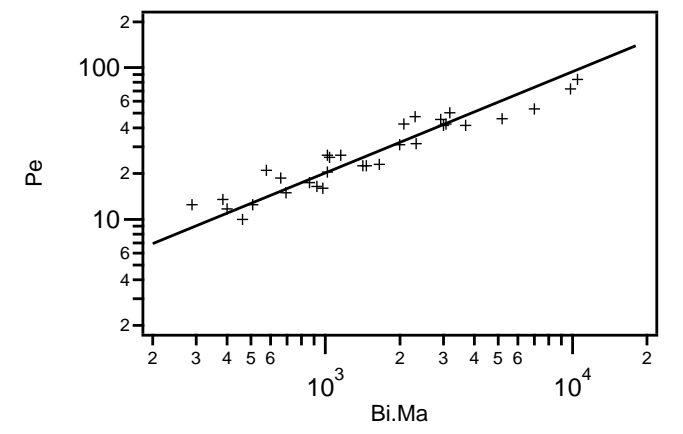

(a)

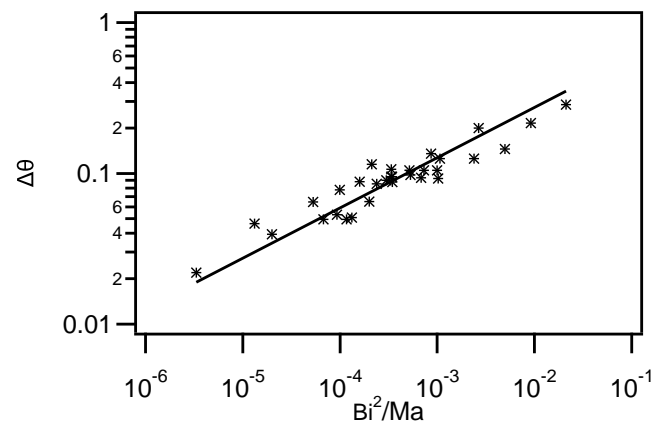

(b)

Fig. .11. Comparison of simulations and correlations in the B domain. (a) $P e$ versus $B i M a$, simulations (crosses) and straight line with slope $2 / 3$, (b) $P e$ versus $B i^{2} / M a$, simulations (stars) and straight line with slope $1 / 3$. 